Results: Each mean age \pm SD of AD and non-AD control was $72.32 \pm 8.66$ and 71.03 \pm 7.75 . Sex ratio of female to male was about $2: 1$. Among the vascular risk factors, DM and PVH was statistically significant factor in AD patients than non-AD patients. The severity of white matter changes was not associated with severity of dementia in AD. Conclusions: DM and PVH had a higher prevalence in AD patients than non-AD patients. Thus, control of DM may reduce the risk of $\mathrm{AD}$ development. $\mathrm{PVH}$ might have several causative factors, and may have some clinical significance of $\mathrm{AD}$, the change itself does not contribute to the progression of $\mathrm{AD}$.

\section{P3-153 SURGERY USING GENERAL ANESTHESIA AND RISK OF DEMENTIA IN THE AGING, DEMOGRAPHICS AND MEMORY STUDY}

Brenda L. Plassman ${ }^{1}$, Kenneth M. Langa ${ }^{2}$, Emily V. A. Finlayson ${ }^{2}$, Mary A. M. Rogers ${ }^{2},{ }^{1}$ Duke University Medical Center, Durham, NC, USA; ${ }^{2}$ University of Michigan, Ann Arbor, MI, USA. Contact e-mail: brenda. plassman@duke.edu

Background: Short-term postoperative cognitive dysfunction is common among the elderly. However, to date there has been minimal evidence to support continued postoperative cognitive decline beyond 6 months. We examined the association between surgery conducted under general anesthesia and risk of dementia in a nationally representative sample in the United States. Methods: We used data from the Aging Demographics and Memory Study (ADAMS), a population-based study of dementia that used a single standardized diagnostic protocol and included subjects from all regions of the country. The ADAMS sample of 856 individuals aged 70 years or older was drawn from participants in the ongoing Health and Retirement Study (HRS). All participants received an extensive in-home clinical and neuropsychological assessment to determine a diagnosis of normal cognition; cognitive impairment, not demented; or dementia. We obtained the full set of Medicare administrative records from 1991-2005 to determine details of each participant's surgical history prior to the outcome date, defined as the estimated age of onset for the demented and the ADAMS assessment date for non-demented individuals. We used proportional hazards models to examine the association between exposure to general anesthesia and the risk of dementia vs. normal cognition. Results: We found that surgery with general anesthesia was associated with increased risk of dementia (Hazard Ratio $(\mathrm{HR})=2.90$; $95 \% \mathrm{CI}=1.70-4.93$ ), independent of the number of hospitalizations prior to the outcome date. This association was evident for non-cardiovascular surgeries $(\mathrm{HR}=3.01 ; 95 \% \mathrm{CI}=1.55-5.82)$ but less so for cardiovascular surgeries $(\mathrm{HR}=2.17 ; 95 \% \mathrm{CI}=0.94-5.04)$. When persons with less than 2 years of Medicare claims and those with questionable cognition at baseline were excluded, the association between general anesthesia and dementia remained $(\mathrm{HR}=2.69 ; 95 \% \mathrm{CI}=1.51-4.80)$. Persons with dementia had 27 hospitalizations per 100 person-years compared to 15 hospitalizations/100 person-years in those with normal cognition $(\mathrm{p}<0.001)$ in the years prior to the outcome date. Conclusions: Our findings suggest an increased risk of dementia after surgery with general anesthesia among older adults. If confirmed, this increased risk for dementia may be an important factor to consider when making decisions about surgery, especially those that are elective, in later life.

\section{P3-154 PROSPECTIVE STUDY OF DIABETES AND COGNITIVE DECLINE AND DEMENTIA SUBTYPES IN CACHE COUNTY, UTAH, USA}

Ronald G. Munger ${ }^{1}$, Jack Charoonruk ${ }^{2}$, JoAnn Tschanz ${ }^{1}$, Peter Zandi ${ }^{3}$, Christopher Corcoran ${ }^{1}$, Heidi Wengreen ${ }^{1}$, Kate Hayden ${ }^{4}$, Maria Norton ${ }^{1}$, Kathleen Welsh-Bohmer ${ }^{4},{ }^{1}$ Utah State University, Logan, UT, USA; ${ }^{2}$ Mahidol University, Bangkok, Thailand; ${ }^{3}$ The Johns Hopkins University, Baltimore, MD, USA; ${ }^{4}$ Duke University, Durham, NC, USA.

Contacte-mail: ron.munger@usu.edu

Background: Type 2 diabetes mellitus (DM) is a well-known cause of vascular disease and cognitive impairment, but its relationship with specific subtypes of dementia, including Alzheimer's disease, is unclear. Methods: The Cache County Memory Study (CCMS) is a prospective study of 5092 men and women aged $65+$ years at baseline in 1995. Cognitive function was assessed with the Modified Mini-Mental State Examination (3MS) at baseline and 3, 7, and 11 years later. Dementia was assessed by clinical examination and standard diagnostic criteria. Diabetes history was self-reported and persons with dementia at baseline were excluded from the analyses. Multivariable mixed effects models were used to evaluate differences in 3MS scores and Cox proportional hazards models were used to evaluate risk of all-cause mild cognitive impairment (MCI), all-cause dementia, and dementia subtypes. Results: DM was associated with a lower mean 3MS score at baseline $(-0.92$ points, $\mathrm{p}<0.01)$ that was maintained over all examination waves. DM was associated with risk of all-cause MCI (hazard ratio (HR) $=1.57$; $95 \%$ confidence interval $(\mathrm{CI})=1.24,1.98)$ and all-cause dementia $(\mathrm{HR}=1.49$, $\mathrm{CI}=1.13,1.97)$. A stronger association was found between DM and the dementia subgroup consisting of $\mathrm{AD}+$ vascular diseases and vascular dementia $(\mathrm{HR}=2.93, \mathrm{CI}=1.94,4.43)$. These results did not differ by sex. No significant overall association was found between DM and risk of AD in the absence of vascular disease $(\mathrm{HR}=1.26, \mathrm{CI}=0.89,1.78)$ however a difference was found by sex with a positive association for men $(\mathrm{HR}=2.25 ; \mathrm{CI}$ $=1.24,4.08)$ and no association for women $(\mathrm{HR}=1.10, \mathrm{CI}=0.63,1.93)$. Conclusions: DM was associated with lower 3MS scores, MCI, all-cause dementia, and dementia with vascular disease in the Cache County Memory Study. AD in the absence of vascular disease was associated with DM in men but not women. DM appears to primarily contribute to age-related cognitive disorders via vascular mechanisms. In the absence of vascular disease, DM may have more subtle neurodegenerative effects via non-vascular mechanisms that vary by sex.

\section{P3-155 CEREBRAL BLOOD FLOW, WHITE MATTER LESIONS AND RISK OF HIPPOCAMPAL ATROPHY: THE SMART-MR STUDY}

Mirjam I. Geerlings, Arnoud J. G. Knoops, Auke P. A. Appelman, Theo D. Witkamp, Yolanda Van Der Graaf, Willem P. T. M. Mali, University Medical Center Utrecht, Utrecht, Netherlands. Contact e-mail: m.geerlings@umcutrecht.nl

Background: Reduced cerebral blood perfusion and white matter lesions (WML) may increase risk for Alzheimer's disease. It has been suggested that particularly the hippocampus is vulnerable to hypoperfusion and ischemia. We investigated whether total parenchymal cerebral blood flow (pCBF) and WML increased risk for hippocampal atrophy in a population of patients with atherosclerotic disease. Methods: Within the SMART-MR (Second Manifestations of ARTerial disease-magnetic resonance) study, a cohort study among patients with a history of atherosclerotic disease, cross-sectional analyses were performed in 392 patients (mean age $62 \pm 9$ years, $84 \%$ male). Automated brain segmentation was used to quantify volumes of WML and total brain on MRI. Using MR angiography, CBF was measured in the internal carotid arteries and basilar artery and was expressed per $100 \mathrm{ml}$ brain volume. Manual volumetric measurements of the hippocampus were performed on a 3-dimensional T1-weighted MRI scan. Volumes of total hippocampus, WML and total brain were expressed relative to intracranial volume. Results: Total mean hippocampal volume was $6.2 \pm 0.7 \mathrm{ml}$. Mean pCBF was $49.8 \pm 11.2 \mathrm{ml} / \mathrm{min}$ per $100 \mathrm{ml}$ brain volume, and median WML volume was $1.26 \mathrm{ml}(10-90 \%$ 0.41-7.41). Linear regression analysis showed that reduced parenchymal CBF was not associated with smaller hippocampal volume after adjustment for age and sex, nor after additional adjustment for vascular risk factors, lacunar infarcts and WML $(\beta=0.02 \mathrm{ml}$ per SD decrease in $\mathrm{pCBF} ; 95 \% \mathrm{CI}-0.05$ to 0.93 ). Higher WML volume was associated with smaller hippocampal volume after adjustment for age, sex, and attenuated but remained statistically significant after additional adjustment for smoking, alcohol use, body mass index, hyperlipidemia, blood pressure, diabetes mellitus and lacunar infarcts $(\beta=-0.08 ; 95 \%$ CI -0.153 to -0.002$)$. However, after adjustment for global brain atrophy, WML were no longer associated with hippocampal volume ( $\beta=-0.06$; $95 \%$ CI -0.127 to 0.018 ). Conclusions: Reduced parenchymal blood flow was not associated with smaller hippocampal volumes in this population. WML were associated with smaller hippocampal volumes, independent of shared vascular risk factors, but there was no evidence of increased hippocampal volume loss compared to other brain tissue. These findings do not suggest that the hippocampus in particular is vulnerable to hypoperfusion and ischemia. 